Scientific Paper

\title{
Study of impacts of different evaluation criteria on gamma pass rates in VMAT QA using MatriXX and EPID
}

\author{
Manthala Padannayil Noufal ${ }^{1,2,3, a}$, Kallikuzhiyil Kochunny Abdullah ${ }^{2,3}$, Puzhakkal Niyas ${ }^{1,2,3}$, Pallimanhayil Abdul \\ Raheem Subha ${ }^{2,3}$ \\ ${ }^{I}$ Department of Medical Physics and Radiotherapy, Baby Memorial Hospital, Calicut, India \\ ${ }^{2}$ Department of Physics, Farook College, Calicut, India \\ ${ }^{3}$ University of Calicut, Malapuram, Kerala, India \\ ${ }^{a}$ E-mail address: noufalsh@gmail.com
}

(received 24 July 2017; revised 4 October 2017; accepted 9 October 2017)

\begin{abstract}
Aim: This study evaluates the impacts of using different evaluation criteria on gamma pass rates in two commercially available QA methods employed for the verification of VMAT plans using different hypothetical planning target volumes (PTVs) and anatomical regions.

Introduction: Volumetric modulated arc therapy (VMAT) is a widely accepted technique to deliver highly conformal treatment in a very efficient manner. As their level of complexity is high in comparison to intensity-modulated radiotherapy (IMRT), the implementation of stringent quality assurance (QA) before treatment delivery is of paramount importance.

Material and Methods: Two sets of VMAT plans were generated using Eclipse planning systems, one with five different complex hypothetical three-dimensional PTVs and one including three anatomical regions. The verification of these plans was performed using a MatriXX ionization chamber array embedded inside a MultiCube phantom and a Varian EPID dosimetric system attached to a Clinac iX. The plans were evaluated based on the $3 \% / 3 \mathrm{~mm}, 2 \% / 2 \mathrm{~mm}$, and $1 \% / 1 \mathrm{~mm}$ global gamma criteria and with three low-dose threshold values $(0 \%, 10 \%$, and $20 \%)$.

Results: The gamma pass rates were above $95 \%$ in all VMAT plans, when the $3 \% / 3 \mathrm{~mm}$ gamma criterion was used and no threshold was applied. In both systems, the pass rates decreased as the criteria become stricter. Higher pass rates were observed when no threshold was applied and they tended to decrease for $10 \%$ and $20 \%$ thresholds.

Conclusion: The results confirm the suitability of the equipments used and the validity of the plans. The study also confirmed that the threshold settings greatly affect the gamma pass rates, especially for lower gamma criteria.
\end{abstract}

Key words: VMAT; quality assurance; MatriXX; EPID; gamma-index method.

\section{Introduction}

Recently, volumetric-modulated arc therapy (VMAT) has become a widely accepted technique for treatment delivery in radiotherapy as it produces highly conformal plans and delivers it in a short time $[1,2]$. VMAT is a complex delivery technique that produces the dose distribution by the real-time variation of three parameters: dose rate, the gantry speed, and the positions of the multi-leaf collimator (MLC) [3]. In intensity-modulated radiotherapy (IMRT), MLC is the only varying parameter; however the level of complexity in VMAT is increased because the gantry speed and the dose rate also change during treatment delivery. The real-time correlation between these parameters is inevitable during VMAT delivery because any variation generates a potential error [4]. The MLC plays a vital role in VMAT delivery and, thus, any error in the MLC position creates an over- or under-dose during treatment [5]. Considerable differences also exist between the optimization processes of VMAT and IMRT, which complicates VMAT plans. In VMAT, the treatment planning system (TPS) uses a series of discrete control points during the optimization process and a continuous real-time interpolation between these control points is required during the delivery [6]. These factors necessitate stringent quality assurance (QA) to be performed before treatment delivery.

Among the several commercially available devices employed for the VMAT QA two-dimensional (2D) arrays are the most widely used. The diode-detector-based MapCHECK (Sun Nuclear Corporation, US) [7], the ion-chamber based Seven29 2D array (PTW, Germany) [8,9], and MatriXX (IBA Dosimetry, Germany) [10-14] are commonly used commercial 2D detector arrays. The MatriXX array has a linear response with dose and it is independent of energy [12]. Additionally, the system provides results comparable with those of the film and various point dose detectors $[15,16]$. However, it has the 
major limitation of angular dependence; specifically, a 7\%$11 \%$ dose discrepancy was reported by Wolfsberger et al. for beam incidence in the perpendicular and oblique directions [11]. This necessitates the application of correction factors, especially in VMAT delivery in which the beam rotates by $360^{\circ}$. The use of correction factors resulted in an improvement in the measurement accuracy of the composite dose verification $[17,18]$. Additionally, some uncertainties have been reported in dose measurements in the low-dose or peripheral regions, which are due to four types of errors: namely, positive bias, over-response to scattered dose, round-off errors, and angular dependence [14].

Another tool used for VMAT QA is the electronic portal imaging device (EPID) [10,19]. EPID as a QA tool is preferred for its large detector density, high contrast, linear response to the dose, and excellent online capabilities. EPID does not require any additional phantoms or cables for QA $[19,20]$. The advantage of EPID over MatriXX is that the resolution limit of the amorphous silicon flat-panel detector of EPID is significantly better than that of the ion chamber detector of MatriXX [21]. However, because the detector in EPID is mounted to a rotating gantry, there is a risk of angledependent detector sag due to the gravitational force, therefore EPID obtains different results for VMAT plans performed using fixed and rotating gantry configurations [10,20]. Despite their many merits, both MatriXX and EPID are unable to measure large-dimension radiation fields because of the smaller sensitive area and lower spatial resolution of both systems compared to those of film dosimetry.

The gamma index $(\gamma)$ is the most widely accepted method for the evaluation of 2D distributions, The measured and calculated $2 \mathrm{D}$ dose distributions in both systems were compared using this method, as recommended by Low et al. [22]. This method combines two important dose comparison criteria: the dose difference $\left(\Delta D_{M}\right)$ and the distance to agreement $\left(\Delta \mathrm{d}_{\mathrm{M}}\right)$. According to this method, when $\gamma \leq 1$, the pixels are regarded as pass points. There different gamma evaluation criteria have been using depending upon institutional protocols. The gamma pass rates can be assessed using the $3 \% / 3 \mathrm{~mm}\left(\Delta \mathrm{D}_{\mathrm{M}}=3 \%\right.$ and $\left.\Delta \mathrm{d}_{\mathrm{M}}=3 \mathrm{~mm}\right), 2 \% / 2 \mathrm{~mm}$ $\left(\Delta \mathrm{D}_{\mathrm{M}}=2 \%\right.$ and $\left.\Delta \mathrm{d}_{\mathrm{M}}=2 \mathrm{~mm}\right)$ and $1 \% / 1 \mathrm{~mm}\left(\Delta \mathrm{D}_{\mathrm{M}}=1 \%\right.$ and $\Delta \mathrm{d}_{\mathrm{M}}=1 \mathrm{~mm}$ ) criteria. Besides that to eliminate the low-dose areas and those outside the field, threshold ( $\mathrm{TH})$ values were set, in combination with gamma criteria and $\mathrm{TH}$ value determine the pass rates. The American Association of Physicists in Medicine (AAPM) Task Group (TG)-119 has recommended the use of either a $10 \%$ dose threshold or a region of interest determined by the jaw setting for $2 \mathrm{D}$ dose analysis. In our clinic, we used the $3 \% / 3 \mathrm{~mm}$ gamma criterion at $\mathrm{TH}_{10 \%}$ for this evaluation and considered gamma pass rates including and above $95 \%$, as acceptable.

The pass rates of gamma analysis highly depend upon its normalization. The normalization of the $\Delta D_{M}$ determines whether the gamma analysis is done locally or globally. In local normalization, $\Delta \mathrm{D}_{\mathrm{M}}$ is calculated by normalizing the percentage difference between the measured dose and calculated dose to the calculated dose at that point. While in global normalization, the $\Delta \mathrm{D}_{\mathrm{M}}$ is normalized to the maximum calculated dose [23]. Although the $3 \% / 3 \mathrm{~mm}$ criterion is the most commonly used condition in, it is not sufficient to detect clinically relevant errors. Hussein et al. reported that small errors introduced in the collimator rotation and the MLC were not detected when the $3 \% / 3 \mathrm{~mm}$ criterion was used and required more stringent gamma evaluation criteria to be detected [9]. Hence, in addition to the $3 \% / 3 \mathrm{~mm}$ criterion, we used the $2 \% / 2 \mathrm{~mm}$ and the $1 \% / 1 \mathrm{~mm}$ criteria for the analysis of VMAT QA. However, the gamma index method is sensitive to the spatial resolution of the measured and calculated dose distribution system [24]. Low and Dempsey [24] recommend the minimum ratio between the spatial resolution of the evaluated distribution and the $\Delta \mathrm{d}_{\mathrm{M}}$ criterion to be $1: 3$. This makes the use of stringent gamma criteria with $\Delta \mathrm{d}_{\mathrm{M}}$ values less than the detector spacing and the calculation grid size a questionable issue. These facts demand a careful approach when using the $1 \% / 1 \mathrm{~mm}$ criterion. It is important to identify the limitations imposed by the combined effects of the gamma index and the equipment in use. The aim of our study is to evaluate the gamma pass rates in VMAT QA, using MatriXX and EPID in planning situations involving complex hypothetical planning target volumes (PTVs) and different clinical conditions, namely, prostate, brain, and head and neck (HN) cases, when different evaluation criteria and TH settings are used.

\section{Materials and Methods}

\subsection{VMAT planning in hypothetical PTVs}

VMAT plans can produce highly conformal dose distributions around a PTV and simultaneously protect the organs at risk (OARs). These distributions are produced by the sophisticated dose optimization algorithms incorporated within the TPS and the plans are generated based on constrains and objectives specified by the user during the optimization process. This is achieved by using MLC modulations of different shapes and sizes, along with the modulation of gantry speed and dose rate. But, even advanced clinical dose calculation algorithms are not able to determine a correct dose distribution for small and irregular MLC patterns where there is lack of charged particle equilibrium, which makes the dosimetry of such patterns a challenge [25]. It is essential to test non clinical plans having different MLC sizes and shapes and with different modulation of the MLC. Evaluation of the VMAT plans using MatriXX and EPID, especially in the above situation, is important to understand the limitation of these devices and how the different MLC shapes affect the quality assurance results. To produce MLC patterns with varying shapes we have randomly selected four complex hypothetical 3D PTVs which resemble the English alphabet letters X (X-PTV), U (U-PTV), Z (Z-PTV) and $\mathrm{O}$ which is a ring shaped PTV (O-PTV) (Figure 1). 
The 2D detector array measuring device has a major drawback of limited spatial resolution. This affects the sensitivity of the detectors to errors and hence, the effectiveness of dose evaluation. So, it is important to know how the spacing of detectors in the device affects the measurement especially when the size of PTV varies. This can be assessed by varying the diameter of the inner and outer rings of O-PTV. Three OPTVs were countered for this purpose. A small O-PTV with inner diameter of $0.25 \mathrm{~cm}$ and outer diameter of $0.5 \mathrm{~cm}(\mathrm{O}$ $\mathrm{PTV}_{0.5 \mathrm{~cm}}$ ), an intermediate-size O-PTV with inner diameter 1 $\mathrm{cm}$ and outer diameter $2 \mathrm{~cm}\left(\mathrm{O}-\mathrm{PTV}_{2 \mathrm{~cm}}\right)$, and a large one with inner and outer diameter of $2 \mathrm{~cm}$ and $4 \mathrm{~cm}$ respectively $(\mathrm{O}-$ $\left.\mathrm{PTV}_{4 \mathrm{~cm}}\right)$.

High organ sparing in VMAT results in a steep dose gradient between the PTV and OAR regions. This produce a large difference between the calculated and the measured dose in highly complex MLC shapes, even for small changes in the position of the dose measuring device [24]. This necessitates testing of the devices in different dose gradient situations. In order to produce different dose gradient regions within the PTV, we have generated a pyramid shaped PTV $(\Delta$-PTV) with a combination of five segmented PTVs with a dose of $80 \mathrm{~Gy}$ (segment-1, red), 60 Gy (segment-2, yellow), 40 Gy (segment3, cyan), 20 Gy (segment-4, brown) and 10 Gy (segment-4, blue) (Figure 2).

All the PTVs were delineated on a homogeneous phantom $(30 \times 30 \times 30 \mathrm{~cm})$ generated using the Eclipse TPS system (Varian Medical Systems, Palo Alto, CA, Version 10). Each of the PTVs is set in coronal plane of size $10 \times 10 \mathrm{~cm}$ and has a height and thickness of $3 \mathrm{~cm}$ each (Figure 1), except in the case of size varying O-PTVs where the thickness is varying in accordance with inner and outer diameter. To control the dose spillage outside the PTVs, and thus generate a highly conformal dose distribution during the plan optimization, three types of avoidance structures (AVSs) were also delineated; namely, $S_{1}$ (green), $S_{2}$ (yellow), and $S_{3}$ (cyan), which formed margins of $1 \mathrm{~cm}, 2 \mathrm{~cm}$, and $3 \mathrm{~cm}$, respectively, around the PTVs (Figure 2).

Two VMAT plans were generated using Eclipse TPS: one with a single arc (gantry angle $179^{\circ}-181^{\circ}$, collimator rotation of $30^{\circ}$ ) and one with double complementary arcs (gantry angles $179^{\circ}-181^{\circ}$ and $181^{\circ}-179^{\circ}$, collimator rotations of $30^{\circ}$ and $\left.330^{\circ}\right)$. The plans were inversely optimized using the progressive resolution optimizer (PRO-II). The hypothetical PTVs (X-PTV, U-PTV, Z-PTV, O-PTV, O-PTV ${ }_{0.5 \mathrm{~cm}}$, O$\mathrm{PTV}_{2 \mathrm{~cm}}$, and O-PTV $4 \mathrm{~cm}$ ) were planned with a dose of $50 \mathrm{~Gy}$ and the maximum dose to the AVS was restricted to $40 \mathrm{~Gy}$, $30 \mathrm{~Gy}$ and $20 \mathrm{~Gy}$ for $\mathrm{S}_{1}, \mathrm{~S}_{2}$, and $\mathrm{S}_{3}$ respectively. The $\Delta$-PTV was optimized for five dose regions: $80 \mathrm{~Gy}, 60 \mathrm{~Gy}, 40 \mathrm{~Gy}$, $20 \mathrm{~Gy}$, and $10 \mathrm{~Gy}$ along with the AVS structures to produce a conformal plan. The optimization was repeated until the desired dose distributions and constrains were achieved. Finally, the doses were calculated using an AAA with a voxel resolution of $0.25 \times 0.25 \times 0.25 \mathrm{~cm}$.
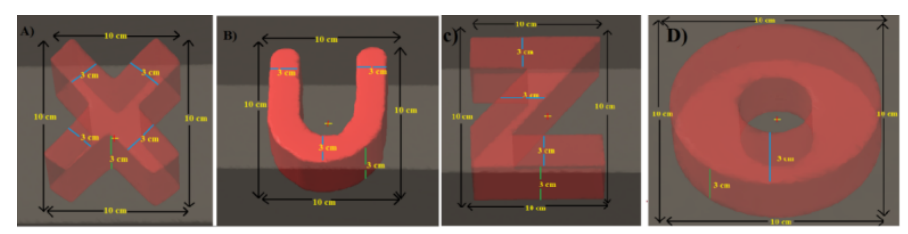

Figure 1. Three dimensional view of the hypothetical PTVs a) XPTV, b) U- PTV, c) Z-PTV, and d) O-PTV on a homogeneous phantom with height (green) and thickness (blue) of $3 \mathrm{~cm}$ each.

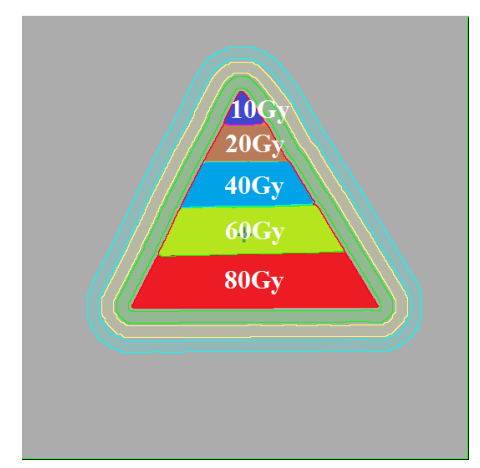

Figure 2. Hypothetical $\Delta$-PTV with five segmented PTVs of thickness $3 \mathrm{~cm}$ and with a dose of $80 \mathrm{~Gy}$ (segment-1, red), $60 \mathrm{~Gy}$ (segment-2, yellow), 40 Gy (segment-3, cyan), 20 Gy (segment-4, brown) and $10 \mathrm{~Gy}$ (segment-4, blue) and avoidance structures $S_{1}$ (green), $S_{2}$ (yellow) and $S_{3}$ (cyan).

\subsection{VMAT planning in patients}

We selected 30 patient plans of three different sites, which included 10 prostate, 10 brain, and $10 \mathrm{HN}$ cancer cases. The prostate cancer patients were treated with $70 \mathrm{~Gy}$ in 28 fractions. The VMAT plans were generated with two complementary full arcs and with collimator rotations of $30^{\circ}$ and $330^{\circ}$. Here, two PTVs were delineated on planning computed tomography (CT): PTV-70Gy for the whole prostate and PTV-50.4Gy for the nodal regions. The plans of the brain tumor patients were created with $60 \mathrm{~Gy}$ in 30 fractions with a single full arc and collimator rotations of $30^{\circ}$. The HN patients were treated with 70 Gy in 33 fractions, with three PTVs, PTV70Gy, PTV-60Gy, and PTV-54Gy, with two complementary full arcs. The VMAT plans were generated using Eclipse, as described in Section 2.1.

\subsection{Verification of plans using MatriXX and EPID}

The pre treatment verification of both the patient plans and the hypothetical PTV-based plans was conducted using two QA systems: a MatriXX system embedded inside a MultiCube phantom (hereafter named IM) and a Varian EPID dosimetric system attached to a Clinac iX (Varian Medical Systems, Palo Alto, CA). All the plans were delivered using the Clinac iX with a Millennium 120-leaf MLC.

The MatriXX is an ionization chamber array consisting of 1020 single air-vented plane-parallel cylindric ionization chambers $(0.55 \mathrm{~cm}$ height, $0.4 \mathrm{~cm}$ diameter, centre-to-centre 
distance $0.76 \mathrm{~cm}$ ) arranged in a $32 \times 32$ matrix (there are no chambers in the corners of the array). A maximum field of view of $24 \times 24 \mathrm{~cm}^{2}$ can be achieved. A The Multicube phantom, in which the MatriXX was embedded, had an $11 \mathrm{~cm}$ thick buildup block and a $7 \mathrm{~cm}$ thick backscatter block. The centre of the MatriXX chamber had a source axis distance of $100 \mathrm{~cm}$ during the measurement. All the VMAT plans were projected onto the CT images of the IM system to generate the verification plans. The array calibration and absolute dose calibration of the ionization chamber were performed using the manufacturer recommended methods. Before each measurement, a 1-h stable time was set and a 10 Gy pre-dose radiation was provided. Background signals were collected for $20 \mathrm{~ms}$ and corrections were performed in the temperature and pressure. The QA was conducted in the planned position with planned gantry angles. A gantry angle sensor was used to detect the gantry positions during VMAT delivery and the corresponding angular correction factors were applied for each measurement. Images of the dose were acquired every $0.5 \mathrm{~s}$ using the movie mode and were then converted into an integral dose distribution. The planned full arcs were independently verified using the IM system. The measured and calculated 2D dose distributions were analysed with the OmniPro-I'mRT (Version 1.7, IBA Dosimetry) analysis software and were subsequently compared using the gamma index method.

The second method for the verification of the plans employed a Varian EPID dosimetric system attached to the Clinac iX (hereafter named EP). The EP system used in our study had amorphous-silicon (aSi 1000) photodiodes arranged in a $40 \times 30 \mathrm{~cm}$ active detector area $(1024 \times 768$ pixels, $0.039 \times 0.034 \mathrm{~cm}$ pixel pitch) [21]. The commissioning of the portal dose image prediction (PDIP) algorithm and the calibration of EPID were performed according to the manufacturer's recommendations. The calibration was conducted at a source to detector distance of $100 \mathrm{~cm}$ for a 6MV beam to achieve the relationship between the calibration unit and monitor units. Each verification plan was generated using the PDIP algorithm in the Eclipse TPS and the 2D dose map was calculated for the planned positions. The measurement was performed in the integration mode with the same geometry and the measured dose distribution was obtained. The comparison between the calculated and measured dose distributions was performed using portal dose analysis software in the Eclipse TPS and was evaluated using the gamma index criteria [22].

\subsection{Evaluation criteria for VMAT QA}

We used the globally normalized gamma pass rates in IM $\left(\mathrm{IM}_{\gamma \%}\right)$ and $\mathrm{EP}\left(\mathrm{EP}_{\gamma \%}\right)$ in our study. The gamma pass rates were assessed using the $3 \% / 3 \mathrm{~mm}\left(\Delta \mathrm{D}_{\mathrm{M}}=3 \%\right.$ and $\left.\Delta \mathrm{d}_{M}=3 \mathrm{~mm}\right)$,
$2 \% / 2 \mathrm{~mm} \quad\left(\Delta \mathrm{D}_{\mathrm{M}}=2 \%\right.$ and $\left.\Delta \mathrm{d}_{\mathrm{M}}=2 \mathrm{~mm}\right)$ and $1 \% / 1 \mathrm{~mm}$ $\left(\Delta \mathrm{D}_{\mathrm{M}}=1 \%\right.$ and $\left.\Delta \mathrm{d}_{\mathrm{M}}=1 \mathrm{~mm}\right)$ criteria. The effect of different $\mathrm{TH}$ values on the gamma pass rates was also assessed. Three TH values were set for each IM and EP measurement. When the $\mathrm{TH}$ was set to zero $\left(\mathrm{TH}_{0 \%}\right)$, all the points in the measured and calculated dose distributions were included in the gamma calculation. However, when the TH was $10 \%\left(\mathrm{TH}_{10 \%}\right)$ and $20 \%$ $\left(\mathrm{TH}_{20 \%}\right)$, the points receiving a dose smaller than $10 \%$ and $20 \%$ of the maximum dose, respectively, were excluded. The same evaluation methods were adopted for both the patient and hypothetical PTV plans. The difference in gamma pass rates between the $\mathrm{TH}_{10 \%}$ and $\mathrm{TH}_{0 \%}\left(\mathrm{TH}_{10-0 \%}\right)$ and $\mathrm{TH}_{10 \%}$ and $\mathrm{TH}_{20 \%}$ $\left(\mathrm{TH}_{10-20 \%}\right)$ were evaluated for the three clinical situations when the $3 \% / 3 \mathrm{~mm}, 2 \% / 2 \mathrm{~mm}$, and $1 \% / 1 \mathrm{~mm}$ gamma criteria were used.

\section{Results}

\subsection{Gamma index analysis of VMAT plans in hypothetical PTVs}

Table 1 shows the mean and standard deviation (SD) in $\mathrm{IM}_{\gamma} \%$ and $\mathrm{EP}_{\gamma \%}$ for the VMAT plans of the four hypothetical PTVs (X-PTV, U-PTV, Z-PTV, and O-PTV). The results for $\mathrm{IM}_{\gamma} \%$ and $\mathrm{EP}_{\gamma \%}$ are comparable in all cases. When the commonly used $3 \% / 3 \mathrm{~mm}$ criterion was considered, the pass rates were above $95 \%$; when $\mathrm{TH}_{20 \%}$ was applied, the Z-PTV exhibited the minimum value, which was $95.9 \% \pm 0.80 \%$ and $96.8 \% \pm$ $0.86 \%$ for IM and EP, respectively. The pass rates were observed to decrease as the criteria became more stringent. Similarly, the pass rates increased for both methods when no threshold $\left(\mathrm{TH}_{0 \%}\right)$ was applied and they tended to decrease for $\mathrm{TH}_{10 \%}$ and $\mathrm{TH}_{20 \%}$. In all cases, the lowest pass rates values were observed for the $1 \% / 1 \mathrm{~mm}$ criteria when a $20 \% \mathrm{TH}$ was applied.

Table 2 summarizes the mean and SD values in $\mathrm{IM}_{\gamma}$ and $\mathrm{EP}_{\gamma \%}$ for the $\Delta$-PTV and for varying sizes of O-PTVs. Among the differently sized O-PTVs, the lowest mean and SD values for the $3 \% / 3 \mathrm{~mm}$ criterion and when $\mathrm{TH}_{20 \%}$ was applied were observed for the O-PTV $0.5 \mathrm{~cm}$; these were $81.0 \% \pm 1.70 \%$ and $84.3 \% \pm 1.15 \%$ for IM and EP respectively, and they were higher in the VMAT plans using O-PTV $2 \mathrm{~cm}$ and O-PTV $4 \mathrm{~cm}$. The $\Delta$-PTV-based VMAT plans also exhibited a reduction in pass rates when changing form $\mathrm{TH}_{0 \%} \quad(97.2 \% \pm 0.95 \%$ and $97.3 \% \pm 1.03 \%)$ to $\mathrm{TH} 20 \%(92.7 \% \pm 2.36 \%$ and $92.0 \% \pm$ $1.25 \%)$. In all cases, it can be observed that the variation in the pass rates when changing from $\mathrm{TH}_{0 \%}$ to $\mathrm{TH}_{20 \%}$ is more evident when the criteria become stringent. 
Table 1. The mean and standard deviation of the gamma pass rates for hypothetical PTV-based (X-PTV, U-PTV, Z-PTV and O-PTV) VMAT plans, as evaluated using IM and EP at different threshold values $\left(\mathbf{T H}_{0 \%}, \mathbf{T H}_{10 \%}\right.$ and $\left.\mathbf{T H}_{20 \%}\right)$.

\begin{tabular}{|c|c|c|c|c|c|c|c|}
\hline VMAT plan & Gamma Criteria & $I_{\text {TH0\% }}$ & $\boldsymbol{E} \boldsymbol{P}_{\text {TH0\% }}$ & $I M_{T H 10 \%}$ & $\boldsymbol{E P}_{\text {TH10\% }}$ & $I M_{T H 20 \%}$ & $\boldsymbol{E P}_{\text {TH20\% }}$ \\
\hline \multirow{3}{*}{ X-PTV } & $3 \% / 3 \mathrm{~mm}$ & $99.3 \pm 0.24$ & $99.5 \pm 0.06$ & $97.6 \pm 0.30$ & $98.3 \pm 0.15$ & $96.4 \pm 0.40$ & $97.2 \pm 0.15$ \\
\hline & $2 \% / 2 \mathrm{~mm}$ & $93.1 \pm 0.99$ & $92.7 \pm 1.53$ & $88.1 \pm 1.20$ & $87.2 \pm 1.05$ & $85.4 \pm 1.40$ & $84.0 \pm 1.05$ \\
\hline & $1 \% / 1 \mathrm{~mm}$ & $72.5 \pm 2.68$ & $74.0 \pm 2.00$ & $53.5 \pm 2.30$ & $55.3 \pm 3.60$ & $49.0 \pm 1.28$ & $52.3 \pm 3.70$ \\
\hline \multirow{3}{*}{ U-PTV } & $3 \% / 3 \mathrm{~mm}$ & $99.2 \pm 0.28$ & $99.5 \pm 0.61$ & $98.4 \pm 0.47$ & $98.1 \pm 0.29$ & $97.1 \pm 0.50$ & $96.9 \pm 0.73$ \\
\hline & $2 \% / 2 \mathrm{~mm}$ & $94.7 \pm 0.51$ & $95.8 \pm 0.72$ & $87.1 \pm 1.20$ & $89.6 \pm 0.55$ & $86.4 \pm 1.31$ & $85.1 \pm 1.20$ \\
\hline & $1 \% / 1 \mathrm{~mm}$ & $76.3 \pm 1.53$ & $77.0 \pm 1.00$ & $56.3 \pm 2.89$ & $58.0 \pm 3.00$ & $54.1 \pm 1.90$ & $53.1 \pm 3.51$ \\
\hline \multirow{3}{*}{ Z-PTV } & $3 \% / 3 \mathrm{~mm}$ & $98.7 \pm 0.21$ & $99.5 \pm 0.40$ & $97.2 \pm 0.23$ & $98.1 \pm 0.12$ & $95.9 \pm 0.80$ & $96.8 \pm 0.86$ \\
\hline & $2 \% / 2 \mathrm{~mm}$ & $93.9 \pm 0.53$ & $95.2 \pm 0.59$ & $91.2 \pm 0.88$ & $89.7 \pm 2.08$ & $85.3 \pm 1.07$ & $86.0 \pm 1.00$ \\
\hline & $1 \% / 1 \mathrm{~mm}$ & $71.3 \pm 1.29$ & $73.7 \pm 1.15$ & $59.2 \pm 3.34$ & $58.0 \pm 1.00$ & $54.8 \pm 2.73$ & $52.7 \pm 2.53$ \\
\hline \multirow{3}{*}{ O-PTV } & $3 \% / 3 \mathrm{~mm}$ & $98.6 \pm 0.88$ & $98.7 \pm 1.53$ & $98.0 \pm 1.28$ & $98.6 \pm 1.46$ & $97.9 \pm 1.29$ & $97.7 \pm 0.58$ \\
\hline & $2 \% / 2 \mathrm{~mm}$ & $92.7 \pm 0.58$ & $92.8 \pm 0.72$ & $90.6 \pm 2.03$ & $89.4 \pm 1.00$ & $88.3 \pm 2.31$ & $87.7 \pm 2.08$ \\
\hline & $1 \% / 1 \mathrm{~mm}$ & $69.0 \pm 1.00$ & $73.0 \pm 1.00$ & $58.3 \pm 1.15$ & $59.7 \pm 1.15$ & $57.0 \pm 2.00$ & $56.6 \pm 1.53$ \\
\hline
\end{tabular}

Table 2. Mean and standard deviation of the gamma pass rates for hypothetical PTV-based $\left(O-P T V_{0.5 \mathrm{~cm}}, O-P T V_{2 \mathrm{~cm}}\right.$, O-PTV VMAT plans, as evaluated using IM and EP at different threshold values $\left(\mathbf{T H}_{0 \%}, \mathbf{T H}_{10 \%}\right.$ and $\left.\mathbf{T H}_{20 \%}\right)$.

\begin{tabular}{cccccccc}
\hline \hline VMAT plan & Gamma Criteria & $\boldsymbol{I M}_{\text {THO\% }}$ & $\boldsymbol{E P}_{\text {THO\% }}$ & $\boldsymbol{I M}_{\text {TH10\% }}$ & $\boldsymbol{E P}_{\text {TH10\% }}$ & $\boldsymbol{I M}_{\text {TH20\% }}$ & $\boldsymbol{E P}_{\text {TH20\% }}$ \\
\hline \multirow{3}{*}{ O-PTV $_{0.5 \mathrm{~cm}}$} & $3 \% / 3 \mathrm{~mm}$ & $98.8 \pm 0.68$ & $98.3 \pm 1.42$ & $90.1 \pm 1.01$ & $92.3 \pm 1.53$ & $81.0 \pm 1.70$ & $84.3 \pm 1.15$ \\
& $2 \% / 2 \mathrm{~mm}$ & $93.1 \pm 0.64$ & $92.1 \pm 0.95$ & $75.1 \pm 1.01$ & $78.0 \pm 1.00$ & $59.4 \pm 0.65$ & $60.3 \pm 1.53$ \\
& $1 \% / 1 \mathrm{~mm}$ & $54.5 \pm 1.11$ & $58.4 \pm 1.53$ & $34.6 \pm 2.17$ & $38.3 \pm 1.10$ & $21.3 \pm 1.60$ & $21.3 \pm 2.08$ \\
\hline \multirow{3}{*}{ O-PTV $_{2 \mathrm{~cm}}$} & $3 \% / 3 \mathrm{~mm}$ & $98.3 \pm 1.16$ & $98.8 \pm 0.98$ & $95.0 \pm 0.42$ & $97.7 \pm 0.58$ & $94.6 \pm 0.85$ & $95.3 \pm 1.13$ \\
& $2 \% / 2 \mathrm{~mm}$ & $95.0 \pm 1.48$ & $96.7 \pm 0.58$ & $87.1 \pm 0.54$ & $88.0 \pm 1.00$ & $76.2 \pm 1.01$ & $78.0 \pm 1.00$ \\
& $1 \% / 1 \mathrm{~mm}$ & $87.2 \pm 1.08$ & $85.8 \pm 2.25$ & $52.3 \pm 1.12$ & $58.0 \pm 1.00$ & $33.7 \pm 0.23$ & $37.7 \pm 0.58$ \\
\hline \multirow{3}{*}{ O-PTV $_{4 \mathrm{~cm}}$} & $3 \% / 3 \mathrm{~mm}$ & $99.5 \pm 0.5$ & $99.0 \pm 1.00$ & $99.3 \pm 0.61$ & $98.3 \pm 1.13$ & $98.5 \pm 1.29$ & $97.7 \pm 2.08$ \\
& $2 \% / 2 \mathrm{~mm}$ & $96.6 \pm 1.00$ & $95.1 \pm 1.80$ & $93.3 \pm 0.60$ & $92.4 \pm 0.58$ & $89.3 \pm 1.20$ & $90.3 \pm 0.58$ \\
& $1 \% / 1 \mathrm{~mm}$ & $91.7 \pm 1.24$ & $92.8 \pm 1.06$ & $66.6 \pm 1.20$ & $68.9 \pm 1.10$ & $66.1 \pm 1.66$ & $65.5 \pm 1.86$ \\
\hline \multirow{3}{*}{$\Delta-\mathrm{PTV}$} & $3 \% / 3 \mathrm{~mm}$ & $97.2 \pm 0.95$ & $97.3 \pm 1.03$ & $94.2 \pm 1.70$ & $94.6 \pm 1.40$ & $92.7 \pm 2.36$ & $92.0 \pm 1.25$ \\
& $2 \% / 2 \mathrm{~mm}$ & $90.7 \pm 1.46$ & $91.7 \pm 1.53$ & $80.9 \pm 2.12$ & $79.2 \pm 0.71$ & $76.1 \pm 3.73$ & $76.7 \pm 1.15$ \\
\hline \hline
\end{tabular}

Table 3. Mean and standard deviation of gamma pass rates for VMAT plans for brain (BR), prostate (PR) and head and neck (HN) cancer patients, obtained using a threshold of $10 \%\left(\mathrm{TH}_{10 \%}\right)$.

\begin{tabular}{|c|c|c|c|c|c|c|}
\hline \multirow{2}{*}{ Gamma Criteria } & \multicolumn{2}{|c|}{ BR } & \multicolumn{2}{|c|}{ PR } & \multicolumn{2}{|c|}{ HN } \\
\hline & IM & EP & IM & EP & IM & EP \\
\hline $3 \% / 3 \mathrm{~mm}$ & $98.3 \pm 0.8$ & $98.5 \pm 0.6$ & $97.7 \pm 1.0$ & $98.0 \pm 0.2$ & $97.2 \pm 0.7$ & $97.5 \pm 2.1$ \\
\hline $2 \% / 2 \mathrm{~mm}$ & $90.8 \pm 2.8$ & $92.4 \pm 1.7$ & $88.1 \pm 2.0$ & $88.5 \pm 3.0$ & $78.9 \pm 4.8$ & $80.6 \pm 8.6$ \\
\hline $1 \% / 1 \mathrm{~mm}$ & $59.0 \pm 4.9$ & $60.6 \pm 3.9$ & $54.6 \pm 4.8$ & $57.5 \pm 4.9$ & $47.7 \pm 8.4$ & $50.7 \pm 7.5$ \\
\hline
\end{tabular}

\subsection{Gamma index analysis of VMAT plans in patients}

The $\mathrm{IM}_{\gamma \%}$ and $\mathrm{EP}_{\gamma \%}$ results were above $95 \%$ in the patientbased VMAT plans when the $3 \% / 3 \mathrm{~mm}$ criterion and a threshold of $\mathrm{TH}_{10 \%}$ were applied (Table 3). However, it must be noted that the gamma pass rates were higher in the brain cancer cases compared to the other two patient categories for all the three evaluation criteria, the maximum values for the $3 \% / 3 \mathrm{~mm}$ criterion were $98.3 \% \pm 0.8 \%$ and $98.5 \% \pm 0.6 \%$ for IM and EP, respectively. The lowest pass rates were exhibited by the $\mathrm{HN}$ cases, which were $97.2 \% \pm 0.7 \%$ and $97.5 \% \pm 2.1 \%$ respectively for IM and EP and for the $3 \% / 3 \mathrm{~mm}$ criterion. These pass rates tended to decline as the criteria became stricter.
Figure 3 depicts the mean and SD for difference in gamma pass rates, calculated using the $3 \% / 3 \mathrm{~mm}, 2 \% / 2 \mathrm{~mm}$ and $1 \% / 1 \mathrm{~mm}$ evaluation criteria in the brain, prostrate, and $\mathrm{HN}$ cancer VMAT plans. The gamma pass rates were observed to be higher when analyzed with $\mathrm{TH}_{0 \%}$ and they decreased when the threshold was increased, in both IM- and EP- based QA systems. The maximum difference in gamma pass rates was observed in the EP-based system for the $\mathrm{HN}$ cases and $\mathrm{TH}_{10-0 \%}$ which were $-1.8 \% \pm 1.6 \%,-9.2 \% \pm 6.3 \%$ and $-12.3 \% \pm 5.3 \%$, respectively for the $3 \% / 3 \mathrm{~mm}, 2 \% / 2 \mathrm{~mm}$, and $1 \% / 1 \mathrm{~mm}$ gamma criteria. When $\mathrm{TH}_{10-20 \%}$ was calculated for the $\mathrm{HN}$ cases, the maximum difference were $1.3 \% \pm 1.1 \%$ in IM and $7.9 \% \pm 6.1 \%$ and $17 \% \pm 4.7 \%$ in $\mathrm{EP}$ for the $3 \% / 3 \mathrm{~mm}$, $2 \% / 2 \mathrm{~mm}$, and $1 \% / 1 \mathrm{~mm}$ gamma criteria. Similarly, in the brain and prostate cancer cases, difference in gamma pass rates were also observed to increase as the gamma criteria become more stringent. 

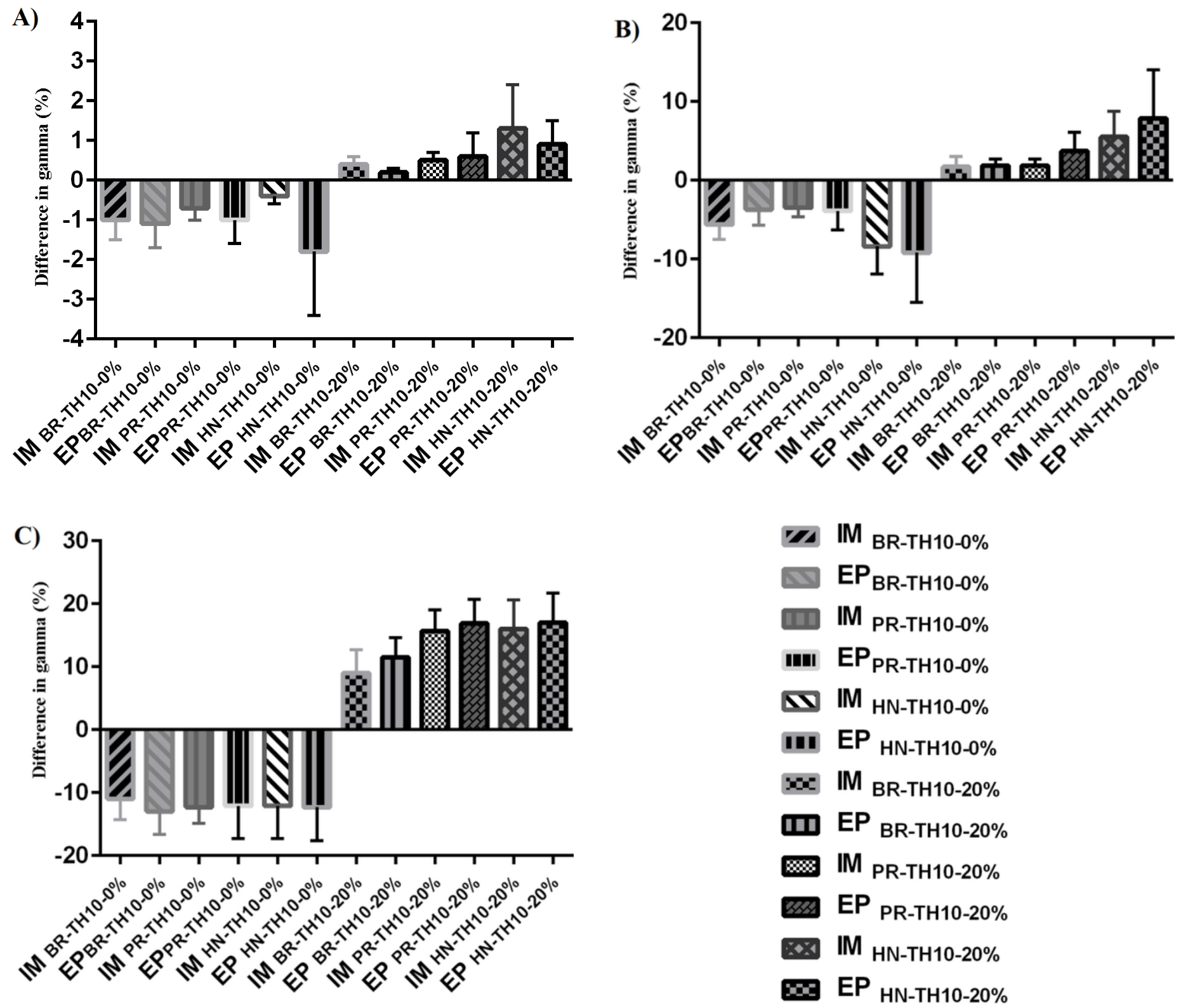

Figure 3. Mean and standard deviation of the percentage differences in gamma pass rates for $\mathbf{T H} \mathbf{H}_{10-0 \%}\left(\mathbf{T H}_{10 \%}-\mathbf{T H}_{\mathbf{0} \%}\right)$ and $\mathbf{T H} \mathbf{H}_{10-20 \%}\left(\mathbf{T H}_{10 \%}\right.$ - TH $_{20 \%}$ ) for brain (BR), prostate (PR) and Head and Neck (HN) clinical plans evaluated using MatriXX (IM) and EPID (EP) at a) $3 \% / 3 \mathrm{~mm}$ b) $2 \% / 2 \mathrm{~mm} \mathrm{c)} 1 \% / 1 \mathrm{~mm}$.

\section{Discussion}

An efficient and consistent testing device has become an essential part of pre treatment verification of highly complicated delivery techniques like VMAT. In our study, we evaluated the effect on gamma pass rates when different gamma evaluation criteria and threshold settings were used, in different hypothetical and clinical situations using the IM and EP verification systems. Different levels of complexity were attained using hypothetical PTVs of different shapes and sizes and by using three different anatomical sites (prostate, brain, and $\mathrm{HN}$ ). Both IM and EP achieved comparable results for the various VMAT plans. The measured and calculated dose distributions showed a good correlation in both systems. Threshold settings were used to define the region of interest of the evaluation field. When no threshold was applied, the gamma calculation considered all the points in the dose plane and obtained a higher pass rate; this effect can mask the fail points inside the field where the PTVs and OARs are present. Therefore, we also investigated the effect of gamma pass rates with $10 \%$ and $20 \%$ thresholds using the $3 \% / 3 \mathrm{~mm}, 2 \% / 2 \mathrm{~mm}$, and $1 \% / 1 \mathrm{~mm}$ gamma criteria.

We observed that as the threshold increased, the pass rates deceased. This reduction was significant when the stringent evaluation criteria $2 \% / 2 \mathrm{~mm}$ and $1 \% / 1 \mathrm{~mm}$ were applied in both the hypothetical and patient-based VMAT plans. Several groups have investigated the applicability of pre treatment verification of the IMRT and VMAT techniques with MatriXX and EPID. Zhu et al. [10] compared the gamma pass rates of 
the VMAT QA using EPID and MatriXX and reported that the gamma pass rates were greater than $98 \%$ when the $3 \% / 3 \mathrm{~mm}$ gamma criterion and a threshold of $10 \%$ were applied. They also noted a reduction in gamma pass rates when the $2 \% / 2 \mathrm{~mm}$ criterion was employed for variable gantry positions. Additionally their results showed higher pass rates in the brain and prostate cancer cases than HN ones. Bailey et al. [26] analyzed the applicability of pre treatment VMAT QA of prostate and HN cancer plans using the Varian EPID and MapCHECK systems and observed gamma pass rates of $98.2 \% \pm 1.65 \%$ and $95.3 \% \pm 5.9 \%$ in the prostate 14 -arc and the HN 12-arc VMAT plans. The outcomes of all these studies are comparable with our results. The lower pass rate exhibited by the HN cases compared to the other two groups is due to the increased level of complexity in the corresponding VMAT plan. This increase in complexity can be attributed to the large dose gradient and the complex dose distribution in the $\mathrm{HN}$ cases.

When the measured and calculated dose distributions are evaluated, there are several sources of uncertainties that must be considered. Positional inaccuracies if any, the detector response, the daily dose variation, and mechanical instabilities of the gantry and collimator of the treatment machine are errors that may modify the gamma pass rates, especially in complex techniques like VMAT [27]. Wagner and Vorwerk observed larger deviations in the gamma pass rates in VMAT plans when the field sizes were very small or very large [27]. This effect was confirmed by our studies of O-PTVs, as significant reductions of the pass points were observed when the size of the PTV was too small owing to resolution of the detector. When the fluence between the two detectors is greatly modulated, the recorded dose is lower than the calculated dose, which also contributes to the dose deviation [18]. We also determined that most of these fail points were near the field boundaries both in the IM and EP QA systems. This is because even small errors in the position of the collimator can result in large dose variations near the edge [18]. During rotation treatment, the angular dependence of MatriXX plays a major role in the gamma pass rates. This dependence was especially increased when the incident beam approached to $90^{\circ}$ in MatriXX. This can be avoided by using gantry angle sensors to determine each gantry position and the corresponding corrections can be applied using the correction factors provided by the OmniPro-I'mRT software. In addition to all these errors, MatriXX overestimates the peripheral dose by $2 \%$ [14].

EPID is a good choice for the verification of plans as it easily achieves accurate positioning and can measure both high- and low-dose gradient regions. Additionally, it exhibits no angular dependence. However, the system has some issues that must be addressed, like electronic disequilibrium produced by insufficient build up material in EPID, inaccuracies in dose measurements created by scattered photons from the phosphor screen, and back scattered radiation from the support arms. Because the position of EPID is fixed with respect to the gantry, it is impossible to asses any errors in the gantry rotation [8]. Furthermore, EPID exhibits a small sag in its position due to gravity; this displacement also contributes to the reduction of pass rates, especially in VMAT plans in which gantry continuously rotates.

The main limitation of $2 \mathrm{D}$ dose verification is that it cannot formulate a correlation between the errors detected during measurement and the dose with the OARs and PTVs. This can be addressed by introducing 3D dose verification methods in clinics. Such methods involve the implementation of an EPID system and a 3D dose reconstruction method by using either a back-projection method [28] or Monte Carlo dose simulations [29]. Furthermore, a technique using the COMPASS system (IBA Dosimetry, Germany) can determine the 3D dose distribution in the patient's anatomy [30]. However, these methods require further clinical investigations and independent commissioning before implementation in clinics. Therefore, the 2D dose verification is still a gold standard for pretreatment plan verification. An action level must be set for the pre treatment QA based on the institution's protocol, the experience of the physicist, and on the recommendation of the AAPM TG -119. The latter propose using 90\% and $88 \%-90 \%$ gamma pass rate for per field and composite 2D dose verification, respectively with the $3 \% / 3 \mathrm{~mm}$ gamma criterion and a $10 \%$ threshold in IMRT. This recommendation is also valid for VMAT plans. The scope of the $3 \mathrm{D}$ analysis in the VMAT plan verification must be investigated in the future and its clinical relevance must be studied in detail.

\section{Conclusions}

We have examined the performance of the MatriXX and EPID systems for QA and verified the validity of VMAT plans. The results obtained for the two systems are comparable in terms of the measured and calculated doses, which confirmed the suitability of the equipments used and the validity of the plans. Our investigation of differently shaped hypothetical PTVs and different clinical situations has provided us with an improved perspective of the plan verification process in different complex situations. Additionally, the study results emphasize that the threshold settings significantly affect the gamma pass rates, especially in the lower gamma criteria; however this effect is reduced in the $3 \% / 3 \mathrm{~mm}$ criterion. Therefore, the threshold value must be selected carefully. 


\section{References}

[1] Verbakel WF, Cuijpers J P, Hoffmans D, et al. Volumetric intensity-modulated arc therapy versus conventional IMRT in head-andneck cancer: a comparative planning and dosimetric study. Int J Radiat Oncol Biol Phys. 2009;74(1):252-259.

[2] Wolff D, Stieler F, Welzel G, et al. Volumetric modulated arc therapy (VMAT) versus serial tomotherapy, step-and-shoot IMRT and 3D-conformal RT for treatment of prostate cancer. Radiother Oncol. 2009;93(2):226-233.

[3] Otto K. Volumetric modulated arc therapy: IMRT in a single gantry arc. Med Phys. 2008;35(1):310-317.

[4] Bedford JL, Warrington AP. Commissioning of volumetric modulated arc therapy (VMAT). Int J Radiat Oncol Biol Phys. 2009;73:537-545.

[5] Tatsumi D, Hosono MN, Nakada R, et al. Direct impact analysis of multi-leaf collimator leaf position errors on dose distributions in volumetric modulated arc therapy: a pass rate calculation between measured planar doses with and without the position errors. Phys Med Biol. 2011;56(20):237-246.

[6] Pardo Montero J, Fenwick JD. The effect of different control point sampling sequences on convergence of VMAT inverse planning Phys Med Biol. 2011;56(8):2569-2583.

[7] Iftimia I, Cirino ET, Xiong L, Mower HW. Quality assurance methodology for Varian RapidArc treatment plans. J Appl Clin Med Phys. 2010;11(4):3164

[8] Chandraraj V, Stathakis S, Manickam R, et al. Consistency and reproducibility of the VMAT plan delivery using three independent validation methods. J Appl Clin Med Phys. 2010;12(1):3373.

[9] Hussein M, Adams EJ, Jordan TJ, et al. A critical evaluation of the PTW 2D-ARRAY seven29 and OCTAVIUS II phantom for IMRT and VMAT verification. J Appl Clin Med Phys. 2013;14(6):4460.

[10] Zhu J, Chen L, Jin G. A comparison of VMAT dosimetric verifications between fixed and rotating gantry positions. Phys Med Biol. 2013;58(15):1315-1322.

[11] Wolfsberger LD, Wagar M, Nitsch P, et al. Angular dose dependence of Matrixx TM and its calibration J Appl Clin Med Phys. 2010;11(1): 241-251.

[12] Herzen J, Todorovic M, Cremers F, et al. Dosimetric evaluation of a 2D pixel ionization chamber for implementation in clinical routine Phys Med Biol. 2007;52(4):1197-1208.

[13] Li JG, Yan G, Liu C. Comparison of two commercial detector arrays for IMRT quality assurance J Appl Clin Med Phys. 2009;10(2):62-74.

[14] Han Z, Ng SK, Bhagwat MS, et al. Evaluation of MatriXX for IMRT and VMAT dose verifications in peripheral dose regions. Med Phys. 2010;37(7):3704-3714.

[15] Wiezorek T, Banz N, Schwedas M, et al. Dosimetric quality assurance for intensity-modulated radiotherapy feasibility study for a filmless approach. Strahlenther Onkol. 2005;181(7):468-474.

[16] Chandraraj V, Stathakis S, Manickam R, et al. Comparison of four commercial devices for RapidArc and sliding window IMRT QA. J Appl Clin Med Phys. 2011;12(2):338-349.

[17] Shimohigashi Y, Araki F, Tominaga H, et al. Angular dependence correction of MatriXX and its application to composite dose verification. J Appl Clin Med Phys. 2012;13(5):81-96.

[18] Boggula R, Birkner M, Lohr F, et al. Evaluation of a 2D detector array for patient-specific VMAT QA with different setups. Phys Med Biol. 2011156(22):7163-7177.

[19] Bakhtiari M, Kumaraswamy L, Bailey DW, et al. Using an EPID for patient-specific VMAT quality assurance Using an EPID for patient-specific VMAT quality assurance. Med Phys. 2011;38(3):1366-73.

[20] Iori M, Cagni E, Paiusco M, et al. Dosimetric verification of IMAT delivery with a conventional EPID system and a commercial portal dose image prediction tool. Med Phys. 2010;37(1):377-390.

[21] Sharma DS, Mhatre V, Heigrujam M, et al. Portal dosimetry for pretreatment verification of IMRT plan : a comparison with 2D ion chamber array. J Appl Clin Med Phys. 2010;11(4):3268.

[22] Low DA, Harms WB, Mutic S, Purdy JA. A technique for the quantitative evaluation of dose distributions. Med Phys. 1998;25(5):656-661.

[23] Bailey DW, Nelms BE, Attwood K, et al. Statistical variability and confidence intervals for planar dose QA pass rates. Med Phys. 2011;38(11):6053-6064.

[24] Low DA, Dempsey JF. Evaluation of the gamma dose distribution comparison method. Med Phys. 2003;30(9):2455-2464.

[25] Das IJ, Ding GX, Ahnesjö A. Small fields: nonequilibrium radiation dosimetry. Med Phys. 2008;35(1):206-215.

[26] Bailey DW, Kumaraswamy L, Bakhtiari M, et al. EPID dosimetry for pretreatment quality assurance with two commercial systems. J Appl Clin Med Phys. 2012;13(4):3736.

[27] Wagner D, Vorwerk H. Two years experience with quality assurance protocol for patient related Rapid Arc treatment plan verification using a two dimensional ionization chamber array. Radiat Oncol. 2011;6(1):21. 
[28] Mans A, Remeijer P, Olaciregui-Ruiz I, et al. 3D dosimetric verification of volumetric-modulated arc therapy by portal dosimetry. Radiother Oncol. 2010;94:181-187.

[29] van Elmpt W, Nijsten S, Dekker AL, et al. Treatment verification in the presence of inhomogeneities using EPID-based three dimensional dose reconstruction Med. Phys. 2007;34(7):2816-2826.

[30] Boggula R, Lorenz F, Mueller L, et al. Experimental validation of a commercial 3D dose verification system for intensity-modulated arc therapies. Phys Med Biol. 2010;55(19):5619-5633.

[31] Mancuso GM, Fontenot JD, Gibbons JP, parker BC. Comparison of action levels for patient-specific quality assurance of intensity modulated radiation therapy and volumetric modulated arc therapy treatments. Med Phys. 2012;39(7):4378-4385. 